\title{
SOME NEW OBSERVATIONS ON THE GÖLLNITZ-GORDON AND ROGERS-RAMANUJAN IDENTITIES
}

\author{
KRISHNASWAMI ALLADI
}

\begin{abstract}
Two new, short and elementary proofs of the Göllnitz-Gordon identities are presented by considering the odd-even split of the Euler Pentagonal Series and the Triangular Series of Gauss. Using this approach the equality of certain shifted partition functions are established. Next, the odd and even parts of the famous Rogers-Ramanujan series are shown to have interesting product representations $(\bmod 80)$. From this, new shifted partition identities are derived.
\end{abstract}

\section{INTRODUCTION}

The celebrated Rogers-Ramanujan identities are

$$
R(q) \doteq \sum_{n=0}^{\infty} \frac{q^{n^{2}}}{(1-q)\left(1-q^{2}\right) \cdots\left(1-q^{n}\right)}=\prod_{m=0}^{\infty} \frac{1}{\left(1-q^{5 m+1}\right)\left(1-q^{5 m+4}\right)},
$$

and

$$
S(q) \doteq \sum_{n=0}^{\infty} \frac{q^{n^{2}+n}}{(1-q)\left(1-q^{2}\right) \cdots\left(1-q^{n}\right)}=\prod_{m=0}^{\infty} \frac{1}{\left(1-q^{5 m+2}\right)\left(1-q^{5 m+3}\right)} .
$$

The identities have a long and rich history (see Andrews [4], Chapter 7 for proof and generalizations). They were first proved by Rogers [10] in 1894 in the second of his now famous trilogy of memoirs on the expansion of infinite products and independently discovered by Ramanujan who communicated them in a letter to Hardy in 1913.

The Göllnitz-Gordon identities are

$$
\begin{aligned}
G(q) & \doteq \sum_{n=0}^{\infty} \frac{q^{n^{2}}(1+q)\left(1+q^{3}\right) \cdots\left(1+q^{2 n-1}\right)}{\left(1-q^{2}\right)\left(1-q^{4}\right) \cdots\left(1-q^{2 n}\right)} \\
& =\prod_{m=0}^{\infty} \frac{1}{\left(1-q^{8 m+1}\right)\left(1-q^{8 m+4}\right)\left(1-q^{8 m+7}\right)},
\end{aligned}
$$

Received by the editors October 30, 1993; presented to the Society on August 15, 1994 (984th AMS meeting).

1991 Mathematics Subject Classification. Primary 05A19, 11P83, 33D15.

Key words and phrases. Göllnitz-Gordon identities, Rogers-Ramanujan identities, odd-even split, shifted partition identities.

Work done at The Pennsylvania State University on sabbatical leave from Florida during 19921993. 
and

$$
\begin{aligned}
H(q) & \doteq \sum_{n=0}^{\infty} \frac{q^{n^{2}+2 n}(1+q)\left(1+q^{3}\right) \cdots\left(1+q^{2 n-1}\right)}{\left(1-q^{2}\right)\left(1-q^{4}\right) \cdots\left(1-q^{2 n}\right)} \\
& =\prod_{m=0}^{\infty} \frac{1}{\left(1-q^{8 m+3}\right)\left(1-q^{8 m+4}\right)\left(1-q^{8 m+5}\right)}
\end{aligned}
$$

Identities (1.3) and (1.4) are equations (36) and (37) in Slater's well-known list of 130 identities of Rogers-Ramanujan type [11]. Gordon [8] and Göllnitz [7] were independently led to these identities combinatorially and while discussing the convergents of a continued fraction. The Göllnitz-Gordon identities are the perfect analogues to the modulus 8 for what the Rogers-Ramanujan identities are to the modulus 5 .

We have several objectives in this paper. First we give two new, short and elementary proofs of the Göllnitz-Gordon identities (see $\S \S 2$ and 3). The simplest proof of (1.3) and (1.4) known so far is due to Andrews [3] making two applications of Jacobi's Triple Product Identity. Our two proofs are simpler and provide connections between the Göllnitz-Gordon identities and certain pivotal identities in the theory of partitions and $q$-series. To be more specific, our first proof (see §2) shows that (1.3) and (1.4) follow from a famous identity of Gauss by doing an even-odd split and applying the Jacobi Triple Product Identity. The second proof (see $\S 3$ ) shows that (1.3) and (1.4) can be obtained by considering the even-odd split in the celebrated Pentagonal Number Theorem of Euler and applying the Quintuple Product Identity.

The ideas underlying these proofs have other interesting applications. Our second objective is to use these ideas to obtain the equality of various shifted partition functions. For example, in $\S 4$ we prove:

Theorem 1. (a) The number of partitions of $2 n+1$ into distinct parts $\equiv$ $\pm 1, \pm 7, \pm 9, \pm 11(\bmod 24)$ is equal to the number of partitions of $2 n$ into distinct parts $\equiv \pm 1, \pm 5, \pm 7, \pm 9(\bmod 24)$.

(b) The number of partitions of $2 n+1$ into distinct parts $\equiv \pm 3, \pm 5, \pm 7, \pm 11$ (mod 24) is equal to the number of partitions of $2 n-1$ into distinct parts $\equiv \pm 1, \pm 3, \pm 5, \pm 11(\bmod 24)$. that

Andrews [5] has discussed several interesting examples of sets $S$ and $T$ such

$$
p_{S}(n)=p_{T}(n-1)
$$

where $p_{S}(n)$ denotes the number of partitions of $n$ whose parts come from the set $S$, repetitions allowed. Note that in Theorem 1 the parts are not allowed to repeat, but it has a companion result, namely,

Theorem 1*. (a) The number of partitions of $n$ into parts $\equiv \pm 1, \pm 6, \pm 7, \pm 8$, $\pm 9, \pm 10, \pm 11, \pm 13, \pm 15, \pm 16, \pm 17, \pm 23(\bmod 48)$ is equal to the number of partitions of $n-1$ into parts $\equiv \pm 1, \pm 5, \pm 6, \pm 7, \pm 8, \pm 9, \pm 15, \pm 16$, $\pm 17, \pm 19, \pm 22, \pm 23(\bmod 48)$.

(b) The number of partitions of $n$ into parts $\equiv \pm 2, \pm 3, \pm 5, \pm 7, \pm 8, \pm 11$, $\pm 13, \pm 16, \pm 17, \pm 18, \pm 19, \pm 21(\bmod 48)$ equals the number of partitions 
of $n-2$ into parts $\equiv \pm 1, \pm 3, \pm 5, \pm 8, \pm 11, \pm 13, \pm 14, \pm 16, \pm 18, \pm 19$, $\pm 21, \pm 23(\bmod 48)$.

Note that Theorem $1^{*}(a)$ is an example of $(1.5)$, whereas Theorem $1^{*}(b)$ is an example of

$$
p_{S}(n)=p_{T}(n-2) \text {. }
$$

Parts (a) of Theorems 1 and $1^{*}$ are related to the first Göllnitz-Gordon identity (1.3), while parts (b) are related to the second identity (1.4). Theorem $1^{*}$ is also proved in $\S 4$. Pursuing ideas in Andrews [5], Kalvade [9] has considered some examples of (1.5), and observed Theorem $1^{*}(a)$; but Theorem $1^{*}(b)$ is new.

The third objective here is to show that the even and odd parts of the RogersRamanujan functions $R(q)$ and $S(q)$ possess beautiful product representations. We were motivated to study the even and odd parts of $R(q)$ and $S(q)$ because our two proofs of (1.3) and (1.4) utilize the even and odd parts of other familiar functions. Given a power series $F(q)$, let

$$
F_{e}(q)=\frac{F(q)+F(-q)}{2} \quad \text { and } \quad F_{o}(q)=\frac{F(q)-F(-q)}{2} .
$$

We then have

$$
\text { Theorem 2. (a) } \begin{aligned}
R_{e}(q)=\prod_{j>0} & \frac{1}{\left(1-q^{j}\right)}, \\
j & \equiv 2(\bmod 4) \text { or } \\
j & \equiv \pm 4, \pm 12, \pm 16, \pm 20, \pm 28, \pm 36(\bmod 80) .
\end{aligned}
$$

(b) $R_{o}(q)=q \prod_{j>0} \frac{1}{\left(1-q^{j}\right)}$,$$
j \equiv 2(\bmod 4) \text { or }
$$$$
j \equiv \pm 4, \pm 8, \pm 20, \pm 24, \pm 32, \pm 36(\bmod 80) \text {. }
$$

(c) $S_{e}(q)=\prod_{j>0} \frac{1}{\left(1-q^{j}\right)}$,

$$
\begin{aligned}
& j \equiv 2(\bmod 4) \text { or } \\
& j \equiv \pm 8, \pm 12, \pm 16, \pm 20, \pm 24, \pm 28(\bmod 80) \text {. }
\end{aligned}
$$

(d) $S_{o}(q)=q^{3} \prod_{j>0} \frac{1}{\left(1-q^{j}\right)}$,

$$
\begin{aligned}
& j \equiv 2(\bmod 4) \text { or } \\
& j \equiv \pm 4, \pm 12, \pm 20, \pm 28, \pm 32, \pm 36(\bmod 80) \text {. }
\end{aligned}
$$

Theorem 2 is proved in $\S 5$. In discussing various properties of $R(q), S(q)$ and other related functions, Watson [12] considered the even and odd parts of the Rogers-Ramanujan functions. In a recent paper [6], Andrews obtained series representations for $R_{e}(q), R_{o}(q), S_{e}(q)$ and $S_{o}(q)$. But the fact that these functions have nice product representations seems to have escaped attention. Indeed from the Andrews series representations the product formulae in Theorem 2 can be obtained by appeal to the Quintuple Product Identity (see remarks at the end of $\S 5$ ). But our proof of Theorem 2 is by a different route; we exploit a modular relation connecting six functions of Rogers which are companions to the Rogers-Ramanujan functions. 
The Andrews series representations have other interesting applications. For instance, from the series for $S_{e}(q)$ and $R_{o}(q)$ we obtain

Theorem 3. (a) The number of partitions of $2 n+1$ into distinct parts $\equiv$ $\pm 1, \pm 9, \pm 11, \pm 13(\bmod 30)$ is equal to the number of partitions of $2 n$ into distinct parts $\equiv \pm 1, \pm 7 \pm 9, \pm 11(\bmod 30)$.

(b) The number of partitions of $2 n+1$ into distinct parts $\equiv \pm 3, \pm 7, \pm 11, \pm 13$ $(\bmod 30)$ is equal to the number of partitions of $2 n-2$ into distinct parts $\equiv \pm 1, \pm 3, \pm 7, \pm 13(\bmod 30)$. ion.

Theorem 3 is to be compared with Theorem 1. It has the following compan-

Theorem 3*. (a) The number of partitions of $n$ into distinct parts $\equiv \pm 1, \pm 9$, $\pm 11, \pm 13(\bmod 30)$ and into parts $\equiv \pm 2, \pm 8, \pm 10, \pm 12(\bmod 30)$ is equal to the number of partitions of $n-1$ into distinct parts $\equiv \pm 1, \pm 7, \pm 9, \pm 11$ $(\bmod 30)$ and into parts $\equiv \pm 2, \pm 8, \pm 10, \pm 12(\bmod 30)$.

(b) The number of partitions of $n$ into distinct parts $\equiv \pm 3, \pm 7, \pm 11, \pm 13$ $(\bmod 30)$ and into parts $\equiv \pm 4, \pm 6, \pm 10, \pm 14(\bmod 30)$ is equal to the number of partitions of $n-3$ into distinct parts $\equiv \pm 1, \pm 3, \pm 7, \pm 13(\bmod 30)$ and into parts $\equiv \pm 4, \pm 6, \pm 10, \pm 14(\bmod 30)$.

Theorems 3 and $3^{*}$ are proved in $\S 6$. Note that unlike Theorem $1^{*}$, in Theorem $3^{*}$ only the even parts are allowed to repeat.

We use the standard notation

$$
(a)_{n}=(a ; q)_{n}=(1-a)(1-a q)\left(1-a q^{2}\right) \ldots\left(1-a q^{n-1}\right)
$$

for a positive integer $n$ and

$$
(a)_{\infty}=\lim _{n \rightarrow \infty}(a)_{n}=\prod_{j=0}^{\infty}\left(1-a q^{j}\right), \quad \text { for }|q|<1 .
$$

Whenever we say $j \equiv b(\bmod m)$ we mean $j>0$ and $j \equiv b(\bmod m)$.

\section{FIRST PROOF OF (1.3) AND (1.4)}

Before giving the proof we note that there are two companion identities to (1.3) and (1.4), namely

$$
\psi_{1}(q) \doteq \sum_{n=0}^{\infty} \frac{q^{2 n^{2}}}{(q)_{2 n}}=\prod_{j \equiv \pm 2, \pm 3, \pm 4, \pm 5(\bmod 16)} \frac{1}{\left(1-q^{j}\right)}
$$

and

$$
\psi_{2}(q) \doteq \sum_{n=0}^{\infty} \frac{q^{2 n^{2}+2 n}}{(q)_{2 n+1}}=\prod_{j \equiv \pm 1, \pm 4, \pm 6, \pm 7(\bmod 16)} \frac{1}{\left(1-q^{j}\right)} .
$$

Identities (2.1) and (2.2) are connected to (1.3) and (1.4) in an elementary way by means of the following transformation:

\section{Lemma.}

$$
\sum_{n=0}^{\infty} \frac{a^{n} q^{n^{2}}\left(-b q ; q^{2}\right)_{n}}{\left(q^{2} ; q^{2}\right)_{n}}=\sum_{n=0}^{\infty} \frac{(a b)^{n} q^{2 n^{2}}\left(-a q^{2 n+1} ; q^{2}\right)_{\infty}}{\left(q^{2} ; q^{2}\right)_{n}}
$$


This lemma can be proved either combinatorially or by expanding the $\left(-b q ; q^{2}\right)_{n}$ term and interchanging the order of summation (see [1]).

Note that when $a=1, b=1$, the left side of the Lemma is $G(q)$. Therefore

$$
G(q)=\sum_{n=0}^{\infty} \frac{q^{n^{2}}\left(-q ; q^{2}\right)_{n}}{\left(q^{2} ; q^{2}\right)_{n}}=\sum_{n=0}^{\infty} \frac{q^{2 n^{2}}\left(-q^{2 n+1} ; q^{2}\right)_{\infty}}{\left(q^{2} ; q^{2}\right)_{n}},
$$

and upon dividing both sides by $\left(-q ; q^{2}\right)_{\infty}$ we get

$$
\frac{G(q)}{\left(-q ; q^{2}\right)_{\infty}}=\sum_{n=0}^{\infty} \frac{q^{2 n^{2}}}{\left(q^{2} ; q^{2}\right)_{n}\left(-q ; q^{2}\right)_{n}} .
$$

We now replace $q$ by $-q$ in the above equation to deduce that

$$
\psi_{1}(q)=\frac{G(-q)}{\left(q ; q^{2}\right)_{\infty}}=G(-q)(-q)_{\infty} .
$$

From (2.3) it follows that (1.3) and (2.1) are equivalent. Similarly, the substitutions $a=q^{2}, b=1$ in the Lemma lead to

$$
\psi_{2}(q)=\frac{H(-q)}{\left(q ; q^{2}\right)_{\infty}}=H(-q)(-q)_{\infty},
$$

which shows that (1.4) and (2.2) are equivalent.

Proof of (1.3) and (1.4). We begin with a famous identity of Gauss which is

$$
g(q)=\sum_{n=0}^{\infty} q^{n(n+1) / 2}=\frac{\left(q^{2} ; q^{2}\right)_{\infty}}{\left(q ; q^{2}\right)_{\infty}}
$$

and compute $g_{e}(q)$ and $g_{0}(q)$ from the series and product in (2.5). More precisely, from the product in (2.5) we get

$$
\begin{aligned}
g_{e}(q) & =\frac{\left(q^{2} ; q^{2}\right)_{\infty}}{2}\left\{\frac{1}{\left(q ; q^{2}\right)_{\infty}}+\frac{1}{\left(-q ; q^{2}\right)_{\infty}}\right\} \\
& =\frac{\left(q^{2} ; q^{2}\right)_{\infty}}{2}\left\{\frac{\left(-q ; q^{2}\right)_{\infty}+\left(q ; q^{2}\right)_{\infty}}{\left(q^{2} ; q^{4}\right)_{\infty}}\right\} \\
& =\frac{\left(q^{4} ; q^{4}\right)_{\infty}}{2}\left\{\sum_{n=0}^{\infty} \frac{q^{n^{2}}}{\left(q^{2} ; q^{2}\right)_{n}}+\sum_{n=0}^{\infty} \frac{(-1)^{n} q^{n^{2}}}{\left(q^{2} ; q^{2}\right)_{n}}\right\} \\
& =\left(q^{4} ; q^{4}\right)_{\infty} \sum_{n=0}^{\infty} \frac{q^{4 n^{2}}}{\left(q^{2} ; q^{2}\right)_{2 n}} \\
& =\left(q^{4} ; q^{4}\right)_{\infty} \psi_{1}\left(q^{2}\right) .
\end{aligned}
$$

On the other hand

$$
\frac{n(n+1)}{2} \equiv 0 \quad(\bmod 2) \Longleftrightarrow n \equiv 0,3(\bmod 4) .
$$


So from (2.7) and the series in (2.5) we get

$$
\begin{aligned}
g_{e}(q) & =\sum_{n=0}^{\infty} q^{8 n^{2}+2 n}+\sum_{n=0}^{\infty} q^{8 n^{2}+14 n+6} \\
& =\sum_{n=0}^{\infty} q^{8 n^{2}+2 n}+\sum_{n=0}^{\infty} q^{8(-1-n)^{2}+2(-1-n)} \\
& =\sum_{n=-\infty}^{\infty} q^{8 n^{2}+2 n} .
\end{aligned}
$$

At this stage we use the Jacobi Triple Product Identity

$$
\sum_{n=-\infty}^{\infty} z^{n} q^{n^{2}}=\left(q^{2} ; q^{2}\right)_{\infty}\left(-z q ; q^{2}\right)_{\infty}\left(-z^{-1} q ; q^{2}\right)_{\infty}
$$

with $q \mapsto q^{8}, z \mapsto q^{2}$ to deduce that the expression in (2.8) is

$$
g_{e}(q)=\left(q^{16} ; q^{16}\right)_{\infty}\left(-q^{6} ; q^{16}\right)_{\infty}\left(-q^{10} ; q^{16}\right)_{\infty}
$$

Having computed $g_{e}(q)$ in two different ways we compare (2.6) and (2.10) to arrive at

$$
\begin{aligned}
\psi_{1}\left(q^{2}\right)=\frac{g_{e}(q)}{\left(q^{4} ; q^{4}\right)_{\infty}} & =\frac{\left(q^{16} ; q^{16}\right)_{\infty}\left(-q^{6} ; q^{16}\right)_{\infty}\left(-q^{10} ; q^{16}\right)_{\infty}}{\left(q^{4} ; q^{4}\right)_{\infty}} \\
& =\prod_{j \equiv \pm 4, \pm 6, \pm 8, \pm 10(\bmod 32)} \frac{1}{\left(1-q^{j}\right)},
\end{aligned}
$$

and from this (2.1) follows upon replacing $q^{2}$ by $q$.

With (2.1) established, (1.3) is only a step away. Indeed by (2.3)

$$
G(-q)=\left(q ; q^{2}\right)_{\infty} \psi_{1}(q)=\frac{\prod_{j \equiv \pm 1, \pm 7(\bmod 16)}\left(1-q^{j}\right)}{\prod_{j \equiv \pm 2, \pm 4(\bmod 16)}\left(1-q^{j}\right)}
$$

or equivalently

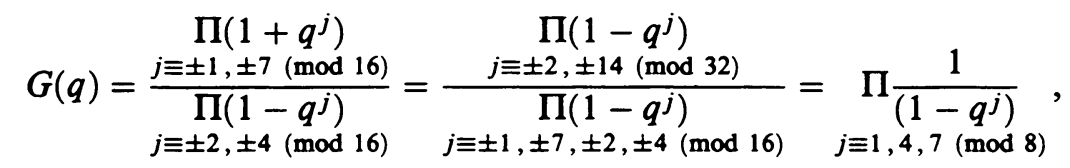

which proves (1.3).

To obtain (1.4) we compute

$$
\begin{aligned}
g_{o}(q) & =\frac{\left(q^{2} ; q^{2}\right)_{\infty}}{2}\left\{\frac{1}{\left(q ; q^{2}\right)_{\infty}}-\frac{1}{\left(-q ; q^{2}\right)_{\infty}}\right\} \\
& =\frac{\left(q^{2} ; q^{2}\right)_{\infty}}{2}\left\{\frac{\left(-q ; q^{2}\right)_{\infty}-\left(q ; q^{2}\right)_{\infty}}{\left(q^{2} ; q^{4}\right)_{\infty}}\right\} \\
& =\frac{\left(q^{4} ; q^{4}\right)_{\infty}}{2}\left\{\sum_{n=0}^{\infty} \frac{q^{n^{2}}}{\left(q^{2} ; q^{2}\right)_{n}}-\sum_{n=0}^{\infty} \frac{(-1)^{n} q^{n^{2}}}{\left(q^{2} ; q^{2}\right)_{n}}\right\} \\
& =\left(q^{4} ; q^{4}\right)_{\infty} \sum_{n=0}^{\infty} \frac{q^{4 n^{2}+4 n+1}}{\left(q^{2} ; q^{2}\right)_{2 n+1}} \\
& =q\left(q^{4} ; q^{4}\right)_{\infty} \psi_{2}\left(q^{2}\right),
\end{aligned}
$$


from the product in (2.5). On the other hand

$$
\frac{n(n+1)}{2} \equiv 1 \quad(\bmod 2) \quad \Longleftrightarrow \quad n \equiv 1,2(\bmod 4),
$$

and so the series in $(2.5)$ yields

$$
\begin{aligned}
g_{o}(q) & =\sum_{n=0}^{\infty} q^{8 n^{2}+6 n+1}+\sum_{n=0}^{\infty} q^{8 n^{2}+10 n+3} \\
& =q\left(\sum_{n=0}^{\infty} q^{8 n^{2}+6 n}+\sum_{n=0}^{\infty} q^{8(-1-n)^{2}+6(-1-n)}\right) \\
& =q \sum_{n=-\infty}^{\infty} q^{8 n^{2}+6 n} .
\end{aligned}
$$

We can now apply the Triple Product Identity (2.9) with $q \mapsto q^{8}, z \mapsto q^{6}$ in (2.14) to obtain

$$
g_{o}(q)=q\left(q^{16} ; q^{16}\right)_{\infty}\left(-q^{2} ; q^{16}\right)_{\infty}\left(-q^{14} ; q^{16}\right)_{\infty}
$$

Upon comparing the two different expressions (2.12) and (2.15) for $g_{o}(q)$ we arrive at

$$
\begin{aligned}
\psi_{2}\left(q^{2}\right) & =\frac{\left(q^{16} ; q^{16}\right)_{\infty}\left(-q^{2} ; q^{16}\right)_{\infty}\left(-q^{14} ; q^{16}\right)_{\infty}}{\left(q^{4} ; q^{4}\right)_{\infty}} \\
& =\underset{j \equiv \pm 2, \pm 8, \pm 12, \pm 14(\bmod 32)}{\Pi \frac{1}{\left(1-q^{j}\right)},},
\end{aligned}
$$

and (2.2) follows from this with $q$ replacing $q^{2}$. From (2.2) the transition to (1.4) via (2.4) is easy and is similar to (2.11).

Remarks. (i) It is interesting that the computation of $g_{e}(q)$ and $g_{o}(q)$ from the product $\left(q^{2} ; q^{2}\right)_{\infty} /\left(q ; q^{2}\right)_{\infty}$ yielded the sums $\psi_{1}$ and $\psi_{2}$, whereas the calculation of $g_{e}(q)$ and $g_{o}(q)$ from the series $\sum q^{n(n+1) / 2}$ let to the product representation.

(ii) Very closely related to (1.3) and (1.4) are the two identities

$$
\begin{aligned}
\sum_{n=0}^{\infty} \frac{q^{n(n+1)}\left(-q^{-1} ; q^{2}\right)_{n}}{\left(q^{2} ; q^{2}\right)_{n}} & =\left(-q ; q^{4}\right)_{\infty}\left(-q^{2} ; q^{4}\right)_{\infty}\left(-q^{4} ; q^{4}\right)_{\infty} \\
& =\frac{1}{\left(q ; q^{8}\right)_{\infty}\left(q^{5} ; q^{8}\right)_{\infty}\left(q^{6} ; q^{8}\right)_{\infty}}
\end{aligned}
$$

and

$$
\begin{aligned}
\sum_{n=0}^{\infty} \frac{q^{n(n+1)}\left(-q ; q^{2}\right)_{n}}{\left(q^{2} ; q^{2}\right)_{n}} & =\left(-q^{3} ; q^{4}\right)_{\infty}\left(-q^{2} ; q^{4}\right)_{\infty}\left(-q^{4} ; q^{4}\right)_{\infty} \\
& =\frac{1}{\left(q^{2} ; q^{8}\right)_{\infty}\left(q^{3} ; q^{8}\right)_{\infty}\left(q^{7} ; q^{8}\right)_{\infty}} .
\end{aligned}
$$

These are immediate consequences of Lebesgue's identity

$$
\sum_{n=0}^{\infty} \frac{q^{n(n+1) / 2}(-b q)_{n}}{(q)_{n}}=\frac{\left(-b q^{2} ; q^{2}\right)_{\infty}}{\left(q ; q^{2}\right)_{\infty}}=\left(-b q^{2} ; q^{2}\right)_{\infty}(-q)_{\infty}
$$


under the substitutions $q \mapsto q^{2}$, and $b \mapsto q^{-3}$ and $b \mapsto q^{-1}$ respectively. Gauss' identity (2.5) is the special case $b=-1$ in (2.18). Thus by deriving the Göllnitz-Gordon identities from (2.5) we see that (1.3), (1.4), (2.16) and (2.17) are all born out of the same source, namely Lebesgue's identity (2.18).

\section{SECOND PROOF OF (1.3) AND (1.4)}

Euler's celebrated Pentagonal Number Theorem is

$$
P(q)=\sum_{n=-\infty}^{\infty}(-1)^{n} q^{\left(3 n^{2}-n\right) / 2}=(q)_{\infty} .
$$

We will compute $P_{e}(q)$ and $P_{o}(q)$ from (3.1) in two different ways. First from the product

$$
\begin{aligned}
P_{e}(q) & =\frac{(q)_{\infty}+(-q ;-q)_{\infty}}{2}=\frac{\left(q^{2} ; q^{2}\right)_{\infty}}{2}\left\{\left(q ; q^{2}\right)_{\infty}+\left(-q ; q^{2}\right)_{\infty}\right\} \\
& =\left(q^{2} ; q^{2}\right)_{\infty} \psi_{1}\left(q^{2}\right),
\end{aligned}
$$

similar to (2.6). Next,

$$
\frac{3 n^{2}-n}{2} \equiv 0 \quad(\bmod 2) \quad \Longleftrightarrow \quad n \equiv 0,3(\bmod 4)
$$

as in (2.7), and so the series in (3.1) yields

$$
P_{e}(q)=\sum_{n=-\infty}^{\infty} q^{24 n^{2}+2 n}-\sum_{n=-\infty}^{\infty} q^{24 n^{2}-14 n+2} .
$$

At this stage we can employ the Quintuple Product Identity

$$
\begin{aligned}
& \sum_{n=-\infty}^{\infty}(-1)^{n} q^{\left(3 n^{2}-n\right) / 2} z^{3 n}\left(1+z q^{n}\right) \\
& \quad=(q)_{\infty}(-z)_{\infty}\left(-z^{-1} q\right)_{\infty}\left(z^{2} q ; q^{2}\right)_{\infty}\left(z^{-2} q ; q^{2}\right)_{\infty}
\end{aligned}
$$

with $q \mapsto q^{16}, z \mapsto-q^{2}$ to deduce that the expression in (3.3) is

$$
P_{e}(q)=\left(q^{16} ; q^{16}\right)_{\infty}\left(q^{2} ; q^{16}\right)_{\infty}\left(q^{14} ; q^{16}\right)_{\infty}\left(q^{12} ; q^{32}\right)_{\infty}\left(q^{20} ; q^{32}\right)_{\infty}
$$

Upon comparing the two different expressions for $P_{e}(q)$ in (3.2) and (3.5) we arrive at

$$
\psi_{1}\left(q^{2}\right)=\frac{\left(q^{16} ; q^{16}\right)_{\infty}\left(q^{2} ; q^{16}\right)_{\infty}\left(q^{14} ; q^{16}\right)_{\infty}\left(q^{12} ; q^{32}\right)_{\infty}\left(q^{20} ; q^{32}\right)_{\infty}}{\left(q^{2} ; q^{2}\right)_{\infty}},
$$

and this is equivalent to (2.1) with $q$ replaced by $q^{2}$. From here the transition to $(1.3)$ via $(2.3)$ is completed as in $\S 2$.

Next, compute $P_{o}(q)$ from the product in (3.1) to get

$$
\begin{aligned}
P_{o}(q) & =\frac{(q)_{\infty}-(-q ;-q)_{\infty}}{2}=\frac{\left(q^{2} ; q^{2}\right)_{\infty}}{2}\left\{\left(q ; q^{2}\right)_{\infty}-\left(-q ; q^{2}\right)_{\infty}\right\} \\
& =-q\left(q^{2} ; q^{2}\right)_{\infty} \psi_{2}\left(q^{2}\right)
\end{aligned}
$$

which is similar to (2.12). On the other hand

$$
\frac{3 n^{2}-n}{2} \equiv 1 \quad(\bmod 2) \quad \Longleftrightarrow \quad n \equiv 1,2(\bmod 4)
$$


as in (2.13), and so the series in (3.1) yields

$$
P_{o}(q)=-q\left\{\sum_{n=-\infty}^{\infty} q^{24 n^{2}+10 n}-\sum_{n=-\infty}^{\infty} q^{24 n^{2}+26 n+6}\right\} .
$$

We may now apply (3.4) with $q \mapsto q^{16}, z \mapsto q^{-6}$ to deduce that the expression in (3.7) is

$$
P_{o}(q)=-q\left(q^{16} ; q^{16}\right)_{\infty}\left(q^{6} ; q^{16}\right)_{\infty}\left(q^{10} ; q^{16}\right)_{\infty}\left(q^{4} ; q^{32}\right)_{\infty}\left(q^{28} ; q^{32}\right)_{\infty}
$$

Finally, comparison of the two different expressions for $P_{o}(q)$ in (3.6) and (3.8) gives

$$
\psi_{2}\left(q^{2}\right)=\frac{\left(q^{16} ; q^{16}\right)_{\infty}\left(q^{6} ; q^{16}\right)_{\infty}\left(q^{10} ; q^{16}\right)_{\infty}\left(q^{4} ; q^{32}\right)_{\infty}\left(q^{28} ; q^{32}\right)_{\infty}}{\left(q^{2} ; q^{2}\right)_{\infty}},
$$

which is equivalent to (2.2) with $q$ replaced by $q^{2}$. From here the transition to $(1.4)$ is easy via $(2.4)$.

Remarks. (i) There are several similarities in these two proofs. As in the previous proof the computation of $P_{e}(q)$ and $P_{o}(q)$ from the product $(q)_{\infty}$ yielded the series $\psi_{1}\left(q^{2}\right)$ and $\psi_{2}\left(q^{2}\right)$ whereas the calculation using the Pentagonal Series gave product representations. Also, both proofs used the companions $\psi_{1}$, $\psi_{2}$ as a means of dealing with $G(q)$ and $H(q)$.

(ii) Computing $P_{e}(q)$ and $P_{o}(q)$ involved a 2-dissection of the Pentagonal Series. More generally, the $k$-dissection of the Pentagonal Series for any integer $k \geq 2$ leads to expressions which can be evaluated as products either by the Quintuple or Triple Product Identities. Although this phenomenon appears to be known to the experts, our utilization of the 2-dissection of $P(q)$ to provide a quick proof of the Göllnitz-Gordon identities is new.

(iii) The decomposition of $P(q)$ considered above is in terms of the even and odd powers of $q$. On the other hand one may decompose the Pentagonal Series in (3.1) in terms of the even and odd values of $n$. We noticed recently [2] that such a split of $P(q)$ leads to a new proof of the Quintuple Product Identity.

In the next section we will use equations (3.3) and (3.7) to prove Theorems 1 and $1^{*}$.

\section{Proof of Theorems 1 and 1 *}

To get the Göllnitz-Gordon identities we employed the Quintuple Product Identity to evaluate the difference of sums in (3.3) and (3.7) as products. To prove Theorem 1 we will use the Triple Product Identity to evaluate each of the four series in (3.3) and (3.7). More precisely, (3.2), (2.9) and (3.3) combine to give

$$
\begin{aligned}
P_{e}(q)= & \psi_{1}\left(q^{2}\right)\left(q^{2} ; q^{2}\right)_{\infty} \\
= & \left(q^{48} ; q^{48}\right)_{\infty}\left(-q^{22} ; q^{48}\right)_{\infty}\left(-q^{26} ; q^{48}\right)_{\infty}-q^{2}\left(q^{48} ; q^{48}\right)_{\infty} \\
& \cdot\left(-q^{10} ; q^{48}\right)_{\infty}\left(-q^{38} ; q^{48}\right)_{\infty}
\end{aligned}
$$

and so

$$
\begin{aligned}
\psi_{1}(q)(q)_{\infty}= & \left(q^{24} ; q^{24}\right)_{\infty}\left(-q^{11} ; q^{24}\right)_{\infty}\left(-q^{13} ; q^{24}\right)_{\infty}-q\left(q^{24} ; q^{24}\right)_{\infty} \\
& \cdot\left(-q^{5} ; q^{24}\right)_{\infty}\left(-q^{19} ; q^{24}\right)_{\infty}
\end{aligned}
$$


upon replacing $q^{2}$ by $q$. Note that by (2.3) the left-hand side of (4.1) is

$$
G(-q)\left(q^{2} ; q^{2}\right)_{\infty}
$$

If we now substitute

$$
G(-q)=\frac{1}{\left(-q ; q^{8}\right)_{\infty}\left(q^{4} ; q^{8}\right)_{\infty}\left(-q^{7} ; q^{8}\right)_{\infty}}
$$

into (4.1) and rearrange the terms suitably, we get

$$
\frac{\left(q^{2} ; q^{2}\right)_{\infty}}{\left(q^{4} ; q^{8}\right)_{\infty}\left(q^{24} ; q^{24}\right)_{\infty}}=\underset{j \equiv \pm 1, \pm 7, \pm 9, \pm 11(\bmod 24)}{\Pi\left(1+q^{j}\right)}-q \underset{j \equiv \pm 1, \pm 5, \pm 7, \pm 9(\bmod 24)}{\Pi\left(1+q^{j}\right)} .
$$

Observe that the left-hand side of (4.2) is an even function of $q$. Therefore the coefficient of $q^{2 n+1}$ is zero. Theorem 1(a) follows by computing the coefficient of $q^{2 n+1}$ from the right-hand side.

To obtain Theorem 1(b) combine (3.6), (2.9) and (3.7) to get

$$
\begin{aligned}
\psi_{2}\left(q^{2}\right)\left(q^{2} ; q^{2}\right)_{\infty}= & \left(q^{48} ; q^{48}\right)_{\infty}\left(-q^{14} ; q^{48}\right)_{\infty}\left(-q^{34} ; q^{48}\right)_{\infty} \\
& -q^{4}\left(q^{48} ; q^{48}\right)_{\infty}\left(-q^{2} ; q^{48}\right)_{\infty}\left(-q^{46} ; q^{48}\right)_{\infty}
\end{aligned}
$$

and this is equivalent to

$$
\begin{aligned}
\psi_{2}(q)(q)_{\infty}= & \left(q^{24} ; q^{24}\right)_{\infty}\left(-q^{7} ; q^{24}\right)_{\infty}\left(-q^{17} ; q^{24}\right)_{\infty} \\
& -q^{2}\left(q^{24} ; q^{24}\right)_{\infty}\left(-q ; q^{24}\right)_{\infty}\left(-q^{23} ; q^{24}\right)_{\infty},
\end{aligned}
$$

upon replacing $q^{2}$ by $q$. By (2.4), the left side of (4.3) is

$$
H(-q)\left(q^{2} ; q^{2}\right)_{\infty}
$$

Substituting

$$
H(-q)=\frac{1}{\left(-q^{3} ; q^{8}\right)_{\infty}\left(q^{4} ; q^{8}\right)_{\infty}\left(-q^{5} ; q^{8}\right)_{\infty}}
$$

into (4.3) and rearranging the terms suitably we get

$$
\frac{\left(q^{2} ; q^{2}\right)_{\infty}}{\left(q^{4} ; q^{8}\right)_{\infty}\left(q^{24} ; q^{24}\right)_{\infty}}=\underset{j \equiv \pm 3, \pm 5, \pm 7, \pm 11(\bmod 24)}{\Pi\left(1+q^{j}\right)}-q^{2} \prod_{j \equiv \pm 1, \pm 3, \pm 5, \pm 11(\bmod 24)}^{\Pi\left(1+q^{j}\right)} .
$$

The left side of (4.4) is an even function of $q$ and so the coefficient of $q^{2 n+1}$ is zero. Theorem 1(b) follows by computing the coefficient of $q^{2 n+1}$ from the right-hand side of (4.4).

To prove Theorem $1^{*}$ replace every factor of the form $1+x$ on the righthand sides of (4.2) and (4.4) by $\left(1-x^{2}\right) /(1-x)$. More precisely, rewrite (4.2) in the form

$$
\frac{\left(q^{2} ; q^{2}\right)_{\infty}}{\left(q^{4} ; q^{8}\right)_{\infty}\left(q^{24} ; q^{24}\right)_{\infty}}=\frac{\underset{j \equiv \pm 2, \pm 14, \pm 18, \pm 22(\bmod 48)}{\Pi\left(1-q^{j}\right)}}{\Pi\left(1-q^{j}\right)}-q \frac{\prod_{\equiv \pm 2, \pm 10, \pm 14, \pm 18(\bmod 48)}}{\Pi\left(1-q^{j}\right)} .
$$


This yields

$$
\begin{gathered}
1=\begin{array}{c}
\Pi \frac{1}{\left(1-q^{j}\right)} \\
-q \equiv \pm 1, \pm 6, \pm 7, \pm 8, \pm 9 ; \pm 10, \pm 11, \pm 13, \pm 15, \pm 16, \pm 17, \pm 23(\bmod 48) \\
\prod \frac{1}{\left(1-q^{j}\right)}
\end{array} . \\
j \equiv \pm 1, \pm 5, \pm 6, \pm 7, \pm 8, \pm 9, \pm 15, \pm 16, \pm 17, \pm 19, \pm 22, \pm 23(\bmod 48)
\end{gathered} .
$$

Theorem $1^{*}$ (a) follows by noting that the coefficient of $q^{n}$ in (4.6) is zero, for $n \geq 1$.

To prove Theorem $1^{*}(\mathrm{~b})$ rewrite $(4.4)$ in the form

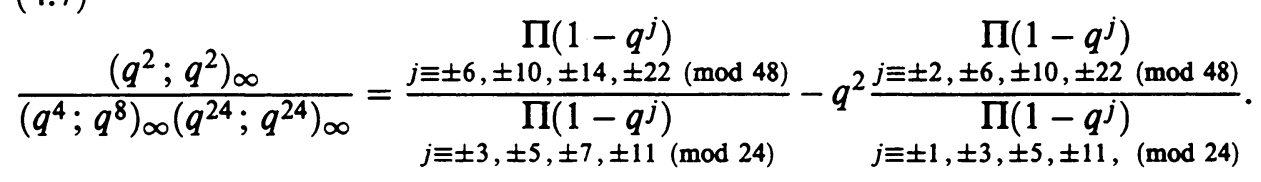

This is equivalent to

$$
\begin{aligned}
& 1=\quad \Pi \frac{1}{\left(1-q^{j}\right)} \\
& j \equiv \pm 2, \pm 3, \pm 5, \pm 7, \pm 8, \pm 11, \pm 13, \pm 16, \pm 17, \pm 18, \pm 19, \pm 21(\bmod 48) \\
& -q^{2} \quad \Pi \frac{1}{\left(1-q^{j}\right)} \\
& j \equiv \pm 1, \pm 3, \pm 5, \pm 8, \pm 11, \pm 13, \pm 14, \pm 16, \pm 18, \pm 19, \pm 21, \pm 23(\bmod 48)
\end{aligned}
$$

Theorem $1^{*}$ (b) follows from (4.8) by observing that the coefficient of $q^{n}$ is zero, for $n \geq 2$.

Remarks. (i) It is worth noting that the left-hand sides of (4.2) and (4.4) are identical. In $\S 6$, while proving Theorem 3 , we will consider a similar pair of identities (see (6.2) and (6.5)) but their left-hand sides, as even functions, are different.

(ii) Another interesting coincidence here is that the factors $\left(q^{4} ; q^{8}\right)_{\infty}$, $\left(q^{24} ; q^{24}\right)_{\infty}$ on the left and those arising from the even integers $j(\bmod 48)$ on the right of (4.5) and (4.7) are all absorbed by $\left(q^{2} ; q^{2}\right)_{\infty}$. This is what leads to (4.6) and (4.8) and to Theorem $1^{*}$. On the other hand, in making the transition from Theorem 3 to Theorem $3^{*}$, we need to impose the restriction that the odd parts do not repeat. For by replacing each factor of the form $(1+x)$ in (6.2) and (6.5) by $\left(1-x^{2}\right) /(1-x)$, all the factors of the form $\left(1-x^{2}\right)$ on the right side are not absorbed by the even function on the left side.

(iii) Instead of rewriting (4.2) and (4.4) in the form (4.5) and (4.7), we may multiply both sides of $(4.2)$ and $(4.4)$ by $\left(q^{4} ; q^{8}\right)_{\infty}\left(q^{24} ; q^{24}\right)_{\infty} /\left(q^{2} ; q^{2}\right)_{\infty}$ and deduce

Theorem $\mathbf{1}^{\prime}$. (a) The number of partitions of $n$ into distinct parts $\equiv \pm 1, \pm 7$, $\pm 9, \pm 11(\bmod 24)$ and into parts $\equiv \pm 2, \pm 6, \pm 8, \pm 10(\bmod 24)$ is equal to the number of partitions of $n$ into distinct parts $\equiv \pm 1, \pm 5, \pm 7, \pm 9(\bmod 24)$ and into parts $\equiv \pm 2, \pm 6, \pm 8, \pm 10(\bmod 24)$.

(b) The number of partitions of $n$ into distinct parts $\equiv \pm 3, \pm 5, \pm 7, \pm 11$ $(\bmod 24)$ and into parts $\equiv \pm 2, \pm 6, \pm 8, \pm 10(\bmod 24)$ is equal to the number 
of partitions of $n-2$ into distinct parts $\equiv \pm 1, \pm 3, \pm 5, \pm 11(\bmod 24)$ and into parts $\equiv \pm 2, \pm 6, \pm 8, \pm 10(\bmod 24)$.

Theorem $1^{\prime}$ is to be compared with Theorem $3^{*}$. But we presented Theorem $1^{*}$ in $\S 1$ because it illustrates the special cancellations that take place in (4.2) and (4.4).

\section{Proof of Theorem 2}

The following six identities due to Rogers [10] are all companions to the Rogers-Ramanujan identities:

$$
\begin{aligned}
& r(q) \doteq \sum_{n=0}^{\infty} \frac{q^{n^{2}}}{\left(q^{4} ; q^{4}\right)_{n}}=\frac{1}{\left(-q^{2} ; q^{2}\right)_{\infty}\left(q ; q^{5}\right)_{\infty}\left(q^{4} ; q^{5}\right)_{\infty}}, \\
& s(q) \doteq \sum_{n=0}^{\infty} \frac{q^{n^{2}+2 n}}{\left(q^{4} ; q^{4}\right)_{n}}=\frac{1}{\left(-q^{2} ; q^{2}\right)_{\infty}\left(q^{2} ; q^{5}\right)_{\infty}\left(q^{3} ; q^{5}\right)_{\infty}}, \\
& \phi_{0}(q) \doteq \sum_{n=0}^{\infty} \frac{q^{n^{2}}}{(q)_{2 n}}=\underset{j \equiv \pm 1, \pm 3, \pm 4, \pm 5, \pm 7, \pm 9(\bmod 20)}{\Pi \frac{1}{\left(1-q^{j}\right)}}, \\
& \phi_{2}(q) \doteq \sum_{n=0}^{\infty} \frac{q^{n^{2}+2 n}}{(q)_{2 n+1}}=\underset{j \equiv \pm 1, \pm 3, \pm 5, \pm 7, \pm 8, \pm 9(\bmod 20)}{\Pi \frac{1}{\left(1-q^{j}\right)}}, \\
& \phi_{1}(q) \doteq \sum_{n=0}^{\infty} \frac{q^{n^{2}+n}}{(q)_{2 n+1}}=\underset{j \equiv \pm 1, \pm 2, \pm 5, \pm 6, \pm 8, \pm 9(\bmod 20)}{\Pi \frac{1}{\left(1-q^{j}\right)}}, \\
& \phi_{3}(q) \doteq \sum_{n=0}^{\infty} \frac{q^{n^{2}+n}}{(q)_{2 n}}=\underset{j \equiv \pm 2, \pm 3, \pm 4, \pm 5, \pm 6, \pm 7(\bmod 20)}{\Pi \frac{1}{\left(1-q^{j}\right)}} .
\end{aligned}
$$

These identities are all in Slater's list [11] and can be derived in an elementary manner from the Rogers-Ramanujan identities.

In November 1990, George Andrews pointed out to me a modular relation connecting these six functions and remarked that this had escaped attention. This modular relation is obtained by splitting the series for $r(q)$ and $s(q)$ into their odd and even subscripts:

$$
r(q)=\sum_{n=0}^{\infty} \frac{q^{4 n^{2}}}{\left(q^{4} ; q^{4}\right)_{2 n}}+\sum_{n=0}^{\infty} \frac{q^{4 n^{2}+4 n+1}}{\left(q^{4} ; q^{4}\right)_{2 n+1}},
$$

and

$$
s(q)=\sum_{n=0}^{\infty} \frac{q^{4 n^{2}+4 n}}{\left(q^{4} ; q^{4}\right)_{2 n}}+\sum_{n=0}^{\infty} \frac{q^{4 n^{2}+8 n+3}}{\left(q^{4} ; q^{4}\right)_{2 n+1}} .
$$

Using (5.3) thru (5.6) we see that these two equations can be rewritten in the form

$$
\begin{aligned}
& r(q)=\phi_{0}\left(q^{4}\right)+q \phi_{1}\left(q^{4}\right), \\
& s(q)=\phi_{3}\left(q^{4}\right)+q^{3} \phi_{2}\left(q^{4}\right) .
\end{aligned}
$$

Previously [1] I had utilized (5.7) and (5.8) to discuss the convergence of a certain continued fraction which is a companion to the famous Rogers-Ramanujan 
continued fraction. For a connection between this continued fraction and Theorem 2 , see $\S 7$.

For the purpose of proving Theorem 2 we rewrite (5.7) and (5.8) in the form

$$
r_{e}(q)=\phi_{0}\left(q^{4}\right), \quad r_{o}(q)=q \phi_{1}\left(q^{4}\right),
$$

and

$$
s_{e}(q)=\phi_{3}\left(q^{4}\right), \quad s_{o}(q)=q^{3} \phi_{2}\left(q^{4}\right) .
$$

But the Rogers functions $r(q)$ and $s(q)$ are connected to the Rogers-Ramanujan functions by the relations

$$
R(q)=r(q)\left(-q^{2} ; q^{2}\right)_{\infty}=\frac{r(q)}{\left(q^{2} ; q^{4}\right)_{\infty}}
$$

and

$$
S(q)=s(q)\left(-q^{2} ; q^{2}\right)_{\infty}=\frac{s(q)}{\left(q^{2} ; q^{4}\right)_{\infty}} .
$$

Now (5.9) and (5.11) yield

$$
R_{e}(q)=\frac{\phi_{0}\left(q^{4}\right)}{\left(q^{2} ; q^{4}\right)_{\infty}}, \quad R_{o}(q)=\frac{q \phi_{1}\left(q^{4}\right)}{\left(q^{2} ; q^{4}\right)_{\infty}},
$$

and

$$
S_{e}(q)=\frac{\phi_{3}\left(q^{4}\right)}{\left(q^{2} ; q^{4}\right)_{\infty}}, \quad S_{o}(q)=\frac{q^{3} \phi_{2}\left(q^{4}\right)}{\left(q^{2} ; q^{4}\right)_{\infty}} .
$$

Note that in (5.13) and (5.14) the $\phi$-functions are evaluated at $q^{4}$ and the residue classes counted in their product representation do not intersect with those counted by $\left(q^{2} ; q^{4}\right)_{\infty}$. Theorem 2 is a consequence of $(5.3),(5.4),(5.5)$, (5.6), (5.13) and (5.14).

Remarks. In a recent paper [6], Andrews has considered the even and odd parts of finite versions of the Rogers-Ramanujan functions. Then, by going to the infinite limit he deduces that

$$
\begin{aligned}
R(q)=\frac{1}{\left(q^{2} ; q^{2}\right)_{\infty}}\{( & \left.\sum_{n=-\infty}^{\infty} q^{60 n^{2}-4 n}-\sum_{n=-\infty}^{\infty} q^{60 n^{2}+44 n+8}\right) \\
& \left.+q\left(\sum_{n=-\infty}^{\infty} q^{60 n^{2}+16 n}-\sum_{n=-\infty}^{\infty} q^{60 n^{2}+64 n+16}\right)\right\},
\end{aligned}
$$

and

$$
\begin{aligned}
S(q)=\frac{1}{\left(q^{2} ; q^{2}\right)_{\infty}}\{( & \left.\sum_{n=-\infty}^{\infty} q^{60 n^{2}+8 n}-\sum_{n=-\infty}^{\infty} q^{60 n^{2}+32 n+4}\right) \\
& \left.+q\left(\sum_{n=-\infty}^{\infty} q^{60 n^{2}+28 n+2}-\sum_{n=-\infty}^{\infty} q^{60 n^{2}+52 n+10}\right)\right\} .
\end{aligned}
$$

Thus in (5.15) and (5.16) the even and odd parts of $R(q)$ and $S(q)$ are expressed in terms of difference of theta series. From here Theorem 2 is only a 
step away if these expressions are rewritten suitably so that the Quintuple Product Identity is applicable. More precisely, rewrite the expression for $R_{e}(q)$ in (5.15) in the form

$$
\frac{1}{\left(q^{2} ; q^{2}\right)_{\infty}}\left(\sum_{n=-\infty}^{\infty} q^{60 n^{2}+4 n}-\sum_{n=-\infty}^{\infty} q^{60 n^{2}+44 n+8}\right)
$$

and apply (3.4) with $q \mapsto q^{40}, z \mapsto-q^{8}$ to deduce that

$$
R_{e}(q)=\left(q^{40} ; q^{40}\right)_{\infty}\left(q^{8} ; q^{40}\right)_{\infty}\left(q^{32} ; q^{40}\right)_{\infty}\left(q^{24} ; q^{80}\right)_{\infty}\left(q^{56} ; q^{80}\right)_{\infty} /\left(q^{2} ; q^{2}\right)_{\infty}
$$

This is equivalent to Theorem 2 (a).

To get Theorem 2(b), replace $n$ by $-n-1$ in the last sum in (5.15) to get

$$
R_{o}(q)=\frac{q}{\left(q^{2} ; q^{2}\right)_{\infty}}\left(\sum_{n=-\infty}^{\infty} q^{60 n^{2}+16 n}-\sum_{n=-\infty}^{\infty} q^{60 n^{2}+56 n+12}\right) .
$$

We may now apply (3.4) with $q \mapsto q^{40}, z \mapsto-q^{12}$ to deduce that

$$
R_{o}(q)=q\left(q^{40} ; q^{40}\right)_{\infty}\left(q^{12} ; q^{40}\right)_{\infty}\left(q^{28} ; q^{40}\right)_{\infty}\left(q^{64} ; q^{80}\right)_{\infty}\left(q^{16} ; q^{80}\right)_{\infty} /\left(q^{2} ; q^{2}\right)_{\infty}
$$

and this is equivalent to Theorem 2 (b).

The derivation of parts (c) and (d) from (5.16) is similar.

\section{Proof of Theorems 3 and $3^{*}$}

In order to prove parts (a) of Theorem 3 and $3^{*}$, we begin with expression for $S_{e}(q)$ given in (5.16) and apply the Triple Product Identity (2.9):

$$
\begin{aligned}
S_{e}(q)=\frac{1}{\left(q^{2} ; q^{2}\right)_{\infty}} & \left\{\sum_{n=-\infty}^{\infty} q^{60 n^{2}+8 n}-\sum_{n=-\infty}^{\infty} q^{60 n^{2}+32 n+4}\right\} \\
=\frac{1}{\left(q^{2} ; q^{2}\right)_{\infty}} & \left\{\left(q^{120} ; q^{120}\right)_{\infty}\left(-q^{52} ; q^{120}\right)_{\infty}\left(-q^{68} ; q^{120}\right)_{\infty}\right. \\
& \left.-q^{4}\left(q^{120} ; q^{120}\right)_{\infty}\left(-q^{28} ; q^{120}\right)_{\infty}\left(-q^{92} ; q^{120}\right)_{\infty}\right\} .
\end{aligned}
$$

Comparing (6.1) with the expression for $S_{e}(q)$ in (5.14) we get

$$
\begin{aligned}
\phi_{3}\left(q^{4}\right)=\frac{1}{\left(q^{4} ; q^{4}\right)_{\infty}}\left\{\left(q^{120} ; q^{120}\right)_{\infty}\left(-q^{52} ; q^{120}\right)_{\infty}\left(-q^{68} ; q^{120}\right)_{\infty}\right. \\
\left.-q^{4}\left(q^{120} ; q^{120}\right)_{\infty}\left(-q^{28} ; q^{120}\right)_{\infty}\left(-q^{92} ; q^{120}\right)_{\infty}\right\} .
\end{aligned}
$$

In the above equation replace $q^{4}$ by $q$ and multiply both sides by $(q)_{\infty}$. Then by $(5.6)$

$$
\begin{aligned}
\phi_{3}(q)(q)_{\infty}= & \left(q^{10} ; q^{10}\right)_{\infty}\left(q ; q^{10}\right)_{\infty}\left(q^{9} ; q^{10}\right)_{\infty}\left(q^{8} ; q^{20}\right)_{\infty}\left(q^{12} ; q^{20}\right)_{\infty} \\
= & \left(q^{30} ; q^{30}\right)_{\infty}\left(-q^{13} ; q^{30}\right)_{\infty}\left(-q^{17} ; q^{30}\right)_{\infty} \\
& -q\left(q^{30} ; q^{30}\right)_{\infty}\left(-q^{7} ; q^{30}\right)_{\infty}\left(-q^{23} ; q^{30}\right)_{\infty} .
\end{aligned}
$$

This may be rewritten in the form

$$
\left(q^{10} ; q^{10}\right)_{\infty}\left(q^{2} ; q^{20}\right)_{\infty}\left(q^{18} ; q^{20}\right)_{\infty}\left(q^{8} ; q^{20}\right)_{\infty}\left(q^{12} ; q^{20}\right)_{\infty} /\left(q^{30} ; q^{30}\right)_{\infty}
$$




$$
\begin{aligned}
= & \left(-q ; q^{10}\right)_{\infty}\left(-q^{9} ; q^{10}\right)_{\infty}\left(-q^{13} ; q^{30}\right)_{\infty}\left(-q^{17} ; q^{30}\right)_{\infty} \\
& -q\left(-q ; q^{10}\right)_{\infty}\left(-q^{9} ; q^{10}\right)_{\infty}\left(-q^{7} ; q^{30}\right)_{\infty}\left(-q^{23} ; q^{30}\right)_{\infty} .
\end{aligned}
$$

Note that the left side of (6.2) is an even function of $q$. Thus the coefficient of $q^{2 n+1}$ is zero. By computing this coefficient from the right side of (6.2), we obtain Theorem 3(a).

Next observe that the left side of $(6.2)$ is equal to the product

$$
\begin{gathered}
\Pi\left(1-q^{j}\right) \\
j \equiv \pm 2, \pm 8, \pm 10, \pm 12(\bmod 30)
\end{gathered} .
$$

Upon dividing both sides of (6.2) by this product we get

$$
\begin{aligned}
& \Pi\left(1+q^{j}\right) \quad \Pi\left(1+q^{j}\right) \\
& 1=\frac{j \equiv \pm 1, \pm 9, \pm 11, \pm 13(\bmod 30)}{\Pi\left(1-q^{j}\right)}-q \frac{j \equiv \pm 1, \pm 7, \pm 9, \pm 11(\bmod 30)}{\Pi\left(1-q^{j}\right)} \text {. }
\end{aligned}
$$

Theorem $3^{*}$ (a) follows by noting that the coefficient of $q^{n}$ in (6.3) is zero for $n \geq 1$.

To prove parts (b) of Theorems 3 and $3^{*}$, start by combining (5.17) and (2.9). This gives

$$
\begin{aligned}
R_{o}(q)=\frac{q}{\left(q^{2} ; q^{2}\right)_{\infty}} & \left\{\left(q^{120} ; q^{120}\right)_{\infty}\left(-q^{44} ; q^{120}\right)_{\infty}\left(-q^{76} ; q^{120}\right)_{\infty}\right. \\
& \left.-q^{12}\left(q^{120} ; q^{120}\right)_{\infty}\left(-q^{4} ; q^{120}\right)_{\infty}\left(-q^{116} ; q^{120}\right)_{\infty}\right\} .
\end{aligned}
$$

Next, compare this with (5.13) to deduce that

$$
\begin{aligned}
\phi_{1}\left(q^{4}\right)=\frac{1}{\left(q^{4} ; q^{4}\right)_{\infty}}\left\{\left(q^{120} ; q^{120}\right)_{\infty}\left(-q^{44} ; q^{120}\right)_{\infty}\left(q^{76} ; q^{120}\right)_{\infty}\right. \\
\left.\quad-q^{12}\left(q^{120} ; q^{120}\right)_{\infty}\left(-q^{4} ; q^{120}\right)_{\infty}\left(-q^{116} ; q^{120}\right)_{\infty}\right\} .
\end{aligned}
$$

In the above equation replace $q^{4}$ by $q$, and multiply both sides by $(q)_{\infty}$. Then (5.5) gives

$$
\begin{aligned}
\phi_{1}(q)(q)_{\infty}= & \left(q^{10} ; q^{10}\right)_{\infty}\left(q^{3} ; q^{10}\right)_{\infty}\left(q^{7} ; q^{10}\right)_{\infty}\left(q^{4} ; q^{20}\right)_{\infty}\left(q^{16} ; q^{20}\right)_{\infty} \\
= & \left(q^{30} ; q^{30}\right)_{\infty}\left(-q^{11} ; q^{30}\right)_{\infty}\left(-q^{19} ; q^{30}\right)_{\infty} \\
& -q^{3}\left(q^{30} ; q^{30}\right)_{\infty}\left(-q ; q^{30}\right)_{\infty}\left(-q^{29} ; q^{30}\right)_{\infty}
\end{aligned}
$$

This may be rewritten in the form

$$
\begin{aligned}
\left(q^{10} ; q^{10}\right)_{\infty}\left(q^{6} ; q^{20}\right)_{\infty}\left(q^{14} ; q^{20}\right)_{\infty}\left(q^{4} ; q^{20}\right)_{\infty}\left(q^{16} ; q^{20}\right)_{\infty} /\left(q^{30} ; q^{30}\right)_{\infty} \\
=\left(-q^{3} ; q^{10}\right)_{\infty}\left(-q^{7} ; q^{10}\right)_{\infty}\left(-q^{11} ; q^{30}\right)_{\infty}\left(-q^{19} ; q^{30}\right)_{\infty} \\
-q^{3}\left(-q^{3} ; q^{10}\right)_{\infty}\left(-q^{7} ; q^{10}\right)_{\infty}\left(-q ; q^{30}\right)_{\infty}\left(-q^{29} ; q^{30}\right)_{\infty}
\end{aligned}
$$

The left side of (6.5) is an even function and so the coefficient of $q^{2 n+1}$ is zero. Computing this coefficient from the right side we get Theorem $3(\mathrm{~b})$.

Finally, note that the left side of (6.5) is

$$
\prod_{j \equiv \pm 4, \pm 6, \pm 10, \pm 14(\bmod 30)}^{\prod} .
$$


Now divide both sides of (6.5) by this product. This yields

$$
\begin{aligned}
& \Pi\left(1+q^{j}\right) \quad \Pi\left(1+q^{j}\right) \\
& 1=\frac{j \equiv \pm 3, \pm 7, \pm 11, \pm 13(\bmod 30)}{\Pi\left(1-q^{j}\right)}-q^{3} \frac{j \equiv \pm 1, \pm 3, \pm 7, \pm 13(\bmod 30)}{\Pi\left(1-q^{j}\right)} \text {. } \\
& j \equiv \pm 4, \pm 6, \pm 10, \pm 14(\bmod 30) \quad j \equiv \pm 4, \pm 6, \pm 10, \pm 14(\bmod 30)
\end{aligned}
$$

Theorem $3^{*}(\mathrm{~b})$ follows by observing that the coefficient of $q^{n}$ in (6.6) is zero, for $n \geq 1$.

Remarks. (i) Unlike (4.2) and (4.4), the even functions on the left of (6.2) and (6.5) are different.

(ii) If we apply the method of this section to the series for $R_{e}(q)$ in (5.15) we get

$$
\begin{aligned}
\left(q^{10} ; q^{10}\right)_{\infty}\left(q^{2} ; q^{10}\right)_{\infty}\left(q^{8} ; q^{10}\right)_{\infty}\left(q^{6} ; q^{20}\right)_{\infty}\left(q^{14} ; q^{20}\right)_{\infty} \\
\quad=\left(q^{30} ; q^{30}\right)_{\infty}\left(-q^{14} ; q^{30}\right)_{\infty}\left(-q^{16} ; q^{30}\right)_{\infty} \\
\quad-q^{2}\left(q^{30} ; q^{30}\right)_{\infty}\left(-q^{4} ; q^{30}\right)_{\infty}\left(-q^{26} ; q^{30}\right)_{\infty} .
\end{aligned}
$$

Similar analysis of $S_{o}(q)$ leads to

$$
\begin{gathered}
\left(q^{10} ; q^{10}\right)_{\infty}\left(q^{4} ; q^{10}\right)_{\infty}\left(q^{6} ; q^{10}\right)_{\infty}\left(q^{2} ; q^{20}\right)_{\infty}\left(q^{18} ; q^{20}\right)_{\infty} \\
\quad=\left(q^{30} ; q^{30}\right)_{\infty}\left(-q^{8} ; q^{30}\right)_{\infty}\left(-q^{22} ; q^{30}\right)_{\infty} \\
\quad-q^{2}\left(q^{30} ; q^{30}\right)_{\infty}\left(-q^{2} ; q^{30}\right)_{\infty}\left(-q^{28} ; q^{30}\right)_{\infty} .
\end{gathered}
$$

Unlike (6.2) and (6.5), identities (6.7) and (6.8) do not yield results like Theorem 3 and $3^{*}$.

\section{CONTINUED FRACTIONS}

Ramanujan viewed (1.1) and (1.2) as arising out of the beautiful continued fraction identity

$$
1+\frac{q}{1+\frac{q^{2}}{1+\frac{q^{3}}{\ddots}}}=\frac{\left(q^{2} ; q^{5}\right)_{\infty}\left(q^{3} ; q^{5}\right)_{\infty}}{\left(q ; q^{5}\right)_{\infty}\left(q^{4} ; q^{5}\right)_{\infty}} .
$$

Indeed the numerator of the above fraction is $R(q)$ and the denominator in $S(q)$; so (7.1) is a consequence of (1.1) and (1.2). Similarly the ratio $G(q) / H(q)$ yields the equally pretty continued fraction identity

$$
1+q+\frac{q^{2}}{1+q^{3}+\frac{q^{4}}{1+q^{5}+\frac{q^{6}}{\ddots}}}=\frac{\left(q^{3} ; q^{8}\right)_{\infty}\left(q^{5} ; q^{8}\right)_{\infty}}{\left(q ; q^{8}\right)_{\infty}\left(q^{7} ; q^{8}\right)_{\infty}} .
$$

There is a nice continued fraction underlying Theorem 2 as well, and we discuss this now.

In a recent paper [1], we established the continued fraction identity

$$
\rho(q) \doteq q+\frac{1}{q^{3}+\frac{1}{q^{5}+\frac{1}{\ddots}}} \stackrel{\text { m.c. }}{=} \frac{\left(q^{2} ; q^{5}\right)_{\infty}\left(q^{3} ; q^{5}\right)_{\infty}}{\left(q ; q^{5}\right)_{\infty}\left(q^{4} ; q^{5}\right)_{\infty}} .
$$


This may be viewed as a companion to Ramanujan's identity (7.1). The letters m.c. in (7.2) mean modified convergence, that is the limit in (7.2) is taken in the following form. Instead of the limit of the ordinary convergents $P_{n}(q) / Q_{n}(q)$, consider the limit as $n \rightarrow \infty$ over the sequence

$$
\frac{P_{0}^{*}(q)}{Q_{0}^{*}(q)}=q+1, \quad \frac{P_{1}^{*}(q)}{Q_{1}^{*}(q)}=q+\frac{1}{q^{3}+1}, \quad \frac{P_{2}^{*}(q)}{Q_{2}^{*}(q)}=q+\frac{1}{q^{3}+\frac{1}{q^{5}+1}}, \ldots
$$

Then the $\operatorname{limit}_{n \rightarrow \infty} P_{n}^{*}(q) / Q_{n}^{*}(q)$ is the product in (7.2).

The continued fraction $\rho(q)$ does not converge in the ordinary sense. The odd convergents $P_{2 n+1}(q) / Q_{2 n+1}(q)$ and the even convergents $P_{2 n}(q) / Q_{2 n}(q)$ both converge but to two different limits. In [1] it was shown that

$$
\begin{aligned}
& \lim _{n \rightarrow \infty} P_{2 n+1}(q)=\phi_{0}\left(q^{4}\right), \\
& \lim _{n \rightarrow \infty} P_{2 n}(q)=q \phi_{1}\left(q^{4}\right), \\
& \lim _{n \rightarrow \infty} Q_{2 n-1}(q)=q^{3} \phi_{2}\left(q^{4}\right), \\
& \lim _{n \rightarrow \infty} Q_{2 n}(q)=\phi_{3}\left(q^{4}\right) .
\end{aligned}
$$

It turns out that

$$
\begin{aligned}
& P_{n}^{*}(q)=P_{n}(q)+Q_{n-1}(q), \\
& Q_{n}^{*}(q)=Q_{n}(q)+Q_{n-1}(q),
\end{aligned}
$$

where $P_{n}^{*}(q)$ and $Q_{n}^{*}(q)$ are defined in (7.3). Thus from (7.4)-(7.9) and the modular equations (5.7) and (5.8) we deduce that

$$
\lim _{n \rightarrow \infty} \frac{P_{n}^{*}(q)}{Q_{n}^{*}(q)}=\frac{\phi_{0}\left(q^{4}\right)+q \phi_{1}\left(q^{4}\right)}{\phi_{0}\left(q^{4}\right)+q^{3} \phi_{2}\left(q^{4}\right)}=\frac{r(q)}{s(q)} .
$$

Thus (7.2) follows from (7.10), (5.1) and (5.2).

We now wish to point out that by (5.13), (5.14) and (7.4)-(7.7)

$$
\lim _{n \rightarrow \infty} \frac{P_{2 n}(q)}{Q_{2 n}(q)}=\frac{R_{o}(q)}{S_{e}(q)}
$$

and

$$
\lim _{n \rightarrow \infty} \frac{P_{2 n+1}(q)}{Q_{2 n+1}(q)}=\frac{R_{e}(q)}{S_{o}(q)} .
$$

Therefore the even and odd parts of the Rogers-Ramanujan functions arise out of the even and odd convergents of the continued fraction $\rho(q)$ and (7.2).

\section{CONCLUDING REMARKS}

In this paper, Theorems $1,1^{*}, 3$ and $3^{*}$, which deal with the equality of various shifted partition functions, were viewed as arising out of the GöllnitzGordon and Rogers-Ramanujan identities. This approach had the advantage that it yielded such results in pairs - that is, these theorems had parts (a) and (b). On the other hand, a simple unified approach to such questions can be given by exploiting connections between the Triple and Quintuple Product Identities, as a consequence of which Theorems $1,1^{*}, 3$ and $3^{*}$ become special cases 
and indeed the minimal examples. The basic ingredients of this approach are already implicit in $\S \S 4$ and 6 . Since this method is of intrinsic interest, it is treated separately [2].

The results of this paper suggest that the following general problem is worthy of investigation: Find examples of sets $S$ such that $F_{e}(q)$ and $F_{o}(q)$ possess nice product representations, where $F(q)$ is the generating function of $p_{S}(n)$. Here, by the product representation being nice, we mean that there are no repeated factors and that factors are either of the form $\left(1-q^{j}\right)^{-1}$ or $\left(1+q^{j}\right)$.

In this connection, Andrews, Garvan and I have recently noticed that the even and odd parts of many such functions $F(q)$ possess nice product representations. Our examples include the Göllnitz-Gordon functions $G(q)$ and $H(q)$, the product $1 /\left(q ; q^{6}\right)_{\infty}\left(q^{5} ; q^{6}\right)_{\infty}$ due to Schur, and certain functions due to Rogers-Selberg to the modulus 7 , among others. One can expect the even and odd parts of many other familiar functions in the theory of partitions and $q$-series to possess interesting product forms. More generally, one can also consider the $k$-dissection of such functions for $k \geq 2$. A systematic discussion of this problem will be taken up elsewhere.

\section{ACKNOWLEDGEMENTS}

I would like to thank the Mathematics Department of The Pennsylvania State University and especially Professor George Andrews for all the support and hospitality extended during my sabbatical visit when this work was done. I also had several stimulating discussions with George Andrews, David Bressoud and Frank Garvan on results related to this paper.

\section{REFERENCES}

1. K. Alladi, On the modified convergence of some continued fractions of Rogers-Ramanujan type, J. Combin. Theory Ser. A 65 (1994), 214-245.

2. K. Alladi, The quintuple product identity and shifted partition functions, (to appear).

3. G. E. Andrews, Partition identities, Adv. Math. 9 (1972), 10-51.

4. - The theory of partitions, Encyclopedia of Math., Vol. 2, Addison Wesley, Reading, MA, 1976.

5. __ Further problems on partitions, Amer. Math. Monthly 94 (1987), 437-439.

6. __ Euler's "Exemplum memorabile inductionis fallacis" and q-trinomial coefficients, J. Amer. Math. Soc. 3 (1990), 653-669.

7. H. Göllnitz, Partitionen mit Differenzenbedingungen, J. Reine Angew. Math. 225 (1967), $154-190$.

8. B. Gordon, Some continued fractions of the Rogers-Ramanujan type, Duke Math J. 32 (1965), 741-748.

9. A. Kalvade, Equality of shifted partition functions, J. Indian Math. Soc. 54 (1989), 155-164.

10. L. J. Rogers, Second memoir on the expansion of certain infinite products, Proc. London Math. Soc. 25 (1894), 318-343.

11. L. J. Slater, Further identities of the Rogers-Ramanujan type, Proc. London Math. Soc. (2) 54 (1952), 147-167.

12. G. N. Watson, Proof of certain identities in combinatory analysis, J. Indian Math. Soc. 20 (1934), 57-69. 